21 Keywords: nanobody, fluorescent proteins, photophysics, nanoboosters, diffusion, brightness,

22 dynamics, protein organisation

\section{Influence of nanobody binding on fluorescence emission, mobility and organization of GFP-tagged proteins}

${ }^{1}$ MRC Human Immunology Unit, Weatherall Institute of Molecular Medicine, University of Oxford, Oxford OX3 9DS, United Kingdom

${ }^{2}$ Institute of Applied Optics and Biophysics, Friedrich-Schiller-University Jena, Max-Wien Platz 4, 07743 Jena, Germany

${ }^{3}$ Leibniz Institute of Photonic Technology e.V., Albert-Einstein-Straße 9, 07745 Jena, Germany

${ }^{5}$ Jena Center of Soft Matters, Friedrich-Schiller-University Jena, Philosophenweg 7, 07743 Jena, Germany

5 Science for Life Laboratory, Department of Women's and Children's Health, Karolinska Institutet, Solna, Sweden

*Correspondence: erdinc.sezgin@ki.se 


\section{Summary}

26 Advanced fluorescence microscopy studies require specific and monovalent molecular labelling

27 with bright and photostable fluorophores. This necessity led to the widespread use of fluorescently

28 labelled nanobodies against commonly employed fluorescent proteins. However, very little is

29 known how these nanobodies influence their target molecules. Here, we observed clear changes

30 of the fluorescence properties, mobility and organisation of green fluorescent protein (GFP) tagged

31 proteins after labelling with an anti-GFP nanobody. Intriguingly, we did not observe any co-

32 diffusion of fluorescently-labelled nanobodies with the GFP-labelled proteins. Our results suggest

33 significant binding of the nanobodies to a non-emissive, oligomerized form of the fluorescent

34 proteins, promoting disassembly into more monomeric forms after binding. Our findings show that

35 great care must be taken when using nanobodies for studying dynamic and quantitative protein

36 organisation.

37

38

39

40

41

42

43

44

45

46

47

48 


\section{Introduction}

Labelling a protein of interest with an antibody is a well-established procedure in molecular biology. Rather large size and multivalence of antibodies, however, limit their application as labelling agents in imaging approaches. Over the past years, the popularity of antigen-binding fragments of antibodies (Fabs) and single-chain nanobodies derived from camelids or shark antibodies grew vastly (Beghein and Gettemans, 2017; Carrington et al., 2019; Leslie, 2018). Both types of molecules are much smaller than full-length antibodies, yet possess similar binding properties to their target proteins (Harmsen and De Haard, 2007; Sahl et al., 2017). Moreover, they only have a single binding site which prevents cross-linking and artificial clustering (Pereira et al., 2019; Sograte-Idrissi et al., 2020; Stanly et al., 2016). Additionally, the stoichiometric labelling of full length antibodies is challenging, whereas fluorescent labelling of a nanobody with 1:1 (nanobody:dye) ratio is regularly achieved (Grußmayer et al., 2014). Nanobodies have successfully been raised against various target molecules and used in microscopy (Pleiner et al., 2015, 2018). Some examples for nanobody epitopes include histones (Jullien et al., 2016), viral protein (Cao et al., 2019), artificial peptides (Braun et al., 2016), clathrin coat components (Traub, 2019), vimentin (Maier et al., 2015) and many more (Aguilar et al., 2019; Mikhaylova et al., 2015). Interestingly, a study using nanobodies targeting synaptic proteins and making use of the nanobodies' smaller size and better penetration capabilities suggested a new pool of synaptic vesicles (Maidorn et al., 2019). The production methods and costs of generating a novel nanobody are comparable to the ones for a standard monoclonal antibody, however, the nanobody can subsequently be produced and harvested from bacteria, yeast or mammalian cell culture and even recombinantly tagged (Arbabi Ghahroudi et al., 1997; Beghein and Gettemans, 2017; Pleiner et al., 2018).

The use of nanobodies in microscopy was fuelled by the development of a green fluorescent protein (GFP) binding nanobody (Ries et al., 2012). GFP or its derivatives (like the enhanced GFP, EGFP) are attractive targets for super-resolution microscopy as they can be considered the biologist's favourite tag, and a GFP-tagged version of a protein of interest is routinely cloned. However, compared to organic dyes, the brightness and photostablity of GFP and its variants is usually worse, limiting its use in some applications. Here, the use of anti-GFP nanobodies labelled with, for example, an organic dye with desired chemical or photophysical properties paved the 
way for a variety of applications (Beghein and Gettemans, 2017; Buser et al., 2018; Fabricius et al., 2018; Farrants et al., 2020; Ries et al., 2012; Sahl et al., 2017) and the development of nanobodies against other fluorescent proteins (FPs) (Platonova et al., 2015). This, in turn, allowed, for example, for multi-colour super-resolution imaging with nanobodies (Sograte-Idrissi et al., 2019).

The binding of the anti-GFP nanobody to GFP has been characterized (Kirchhofer et al., 2010; Klamecka et al., 2015; Della Pia and Martinez, 2015) and it has already been noted that the binding of a nanobody to a fluorescent protein can change the photophysical properties of GFP such as fluorescence brightness, depending on the binding site (Kirchhofer et al., 2010). This influence has been exploited for in vivo studies (Llama Tags in fruit fly embryo (Bothma et al., 2018)). General fluorescence properties of GFP have been studied in depth (Conyard et al., 2011; Jung et populations have been shown to depend on environmental characteristics such as solvent properties (e.g. pH, viscosity), illumination intensity, and wavelength (Ghosh et al., 2017; Jung et al., 2005; Lippincott-Schwartz and Patterson, 2009; Niwa et al., 1996; Tsien, 1998).

Influences of the nanobody on the functionality of the FP-tagged protein have been indicated before (Küey et al., 2019), and we here present new insights by investigating effects of nanobody binding on the fluorescence emission, organization and mobility of GFP-tagged proteins. Specifically, we used commercially available unlabelled and fluorescently labelled GFP-binding nanobodies $(\mathrm{Nb})$ in combination with fluorescence imaging and spectroscopic tools such as fluorescence correlation spectroscopy (FCS) for GFP and EGFP in solution, attached to synthetic membranes, and expressed on the surface of live cells as (E)GFP-tagged GPI-anchored proteins. Our data suggests that the anti-GFP $\mathrm{Nb}$ binds a dark oligomeric form of GFP and promotes reorganization by releasing bright monomers.

\section{Results and Discussion}

\section{Nanobody binding in solution}

We first investigated the basic fluorescence properties of GFP and EGFP before and after addition

107 of unlabelled nanobody $(\mathrm{Nb})$ in solution. Specifically, using a fluorescence spectrometer we 108 investigated changes in total fluorescence intensity and fluorescence spectra for recombinant his- 
109 tagged (E)GFP in PBS (pH 7.4, room temperature). Figure 1 shows the respective excitation and 110 emission spectra. As reported before (Tsien, 1998), we found two excitation peaks at around 395

$111 \mathrm{~nm}$ and $480 \mathrm{~nm}$ for GFP corresponding to the neutral and deprotonated (anionic) state of the

112 fluorochrome, respectively (Chattoraj et al., 1996; Chudakov and Lukyanov, 2003), and one

113 excitation peak at around $480 \mathrm{~nm}$ for EGFP (Figure 1a,b). The anionic state is usually considered

114 for fluorescence microscopy experiments, using a standard $488 \mathrm{~nm}$ laser line for excitation. Due

115 to the requirement for UV excitation, the neutral form of GFP is less used. Interestingly and already

116 previously indicated (Kirchhofer et al., 2010), Nb binding promotes anionic state absorption,

117 revealed by $\mathrm{a} \approx 2$-fold reduction of the excitation peak at $390 \mathrm{~nm}$ and a corresponding $\approx 3$-fold

118 increase at $480 \mathrm{~nm}$ for GFP (Figure 1a). Similarly but less pronounced, the excitation peak at

119 around $480 \mathrm{~nm}$ also increased by $25 \%$ for EGFP upon interaction with the $\mathrm{Nb}$ (Figure $1 \mathrm{~b}$ ). The

$120 \mathrm{Nb}$ binding did not induce any shifts in the emission spectra of GFP or EGFP (excited with 488

$121 \mathrm{~nm}$, Figure 1c,d), also not under UV excitation (405 nm excitation, Figure 1e,f), i.e. peak positions

122 of the spectra remained the same with inefficient excitation of EGFP at $405 \mathrm{~nm}$. Overall, in solution

123 GFP and EGFP experience $\mathrm{a} \approx 3.5-$ and $\approx 1.5$-fold increase in total integrated fluorescence

124 emission (510 $\mathrm{nm}$ to $600 \mathrm{~nm}$ ) induced by the $\mathrm{Nb}$ binding, apparently mainly due to the increase in 125 absorption at around $480 \mathrm{~nm}$. 

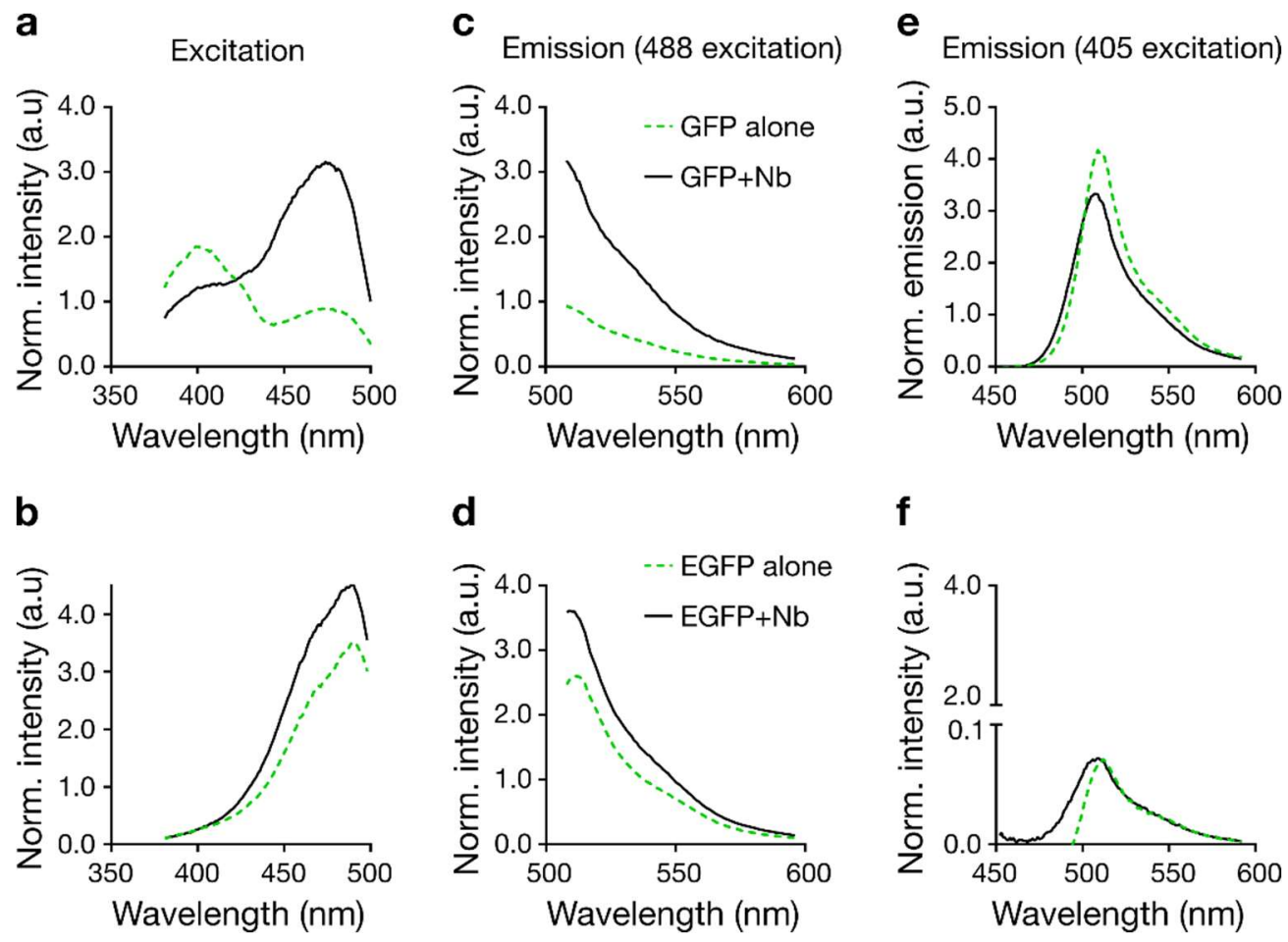

Figure 1: Change in excitation and emission spectra of recombinant GFP and EGFP in solution upon

addition of unlabelled $\mathrm{Nb}$. Excitation spectra for fluorescence detection at 510 - $520 \mathrm{~nm}(\mathrm{a}, \mathrm{b})$ and emission spectra following $488 \mathrm{~nm}(\mathrm{c}, \mathrm{d})$ and $405 \mathrm{~nm}$ excitation (e, f) of GFP-His (a,c,e) and EGFP-HIs (b,d, f) without $\mathrm{Nb}$ (green dashed line) and with unlabelled $\mathrm{Nb}$ (solid black line). All spectra are averages of three measurements.

\section{Nanobody binding on GUV membranes}

In cell biology and microscopy, antibodies and nanobodies are commonly used to investigate the spatial organisation of membrane proteins. Therefore, we next tested changes in fluorescence properties of (E)GFP fluorescence at lipid membranes upon binding of unlabelled $\mathrm{Nb}$. We first chose controlled conditions, employing GFP and EGFP attached to synthetic membranes of giant

138 unilamellar vesicles (GUVs, made of dipalmitoylphosphatidylcholine (DOPC) lipid) via a His-tag (using

DGS-NTA

(1,2-dioleoyl-sn-glycero-3-[(N-(5-amino-1-carboxypentyl)iminodiacetic acid)succinyl])). For both GFP and EGFP, we observed an increase in total fluorescence intensity upon addition of unlabelled $\mathrm{Nb}$, slightly higher $(\approx 2-4$ fold $)$ than in solution and with slight differences between GFP and EGFP (4-fold compared to 2.5-fold, respectively) (Figure 2a,b).

To decipher this slight difference in increase in fluorescence intensity further, we tested how the

144 fluorescence emission per individual GFP and EGFP molecule changed upon Nb binding. For this, 

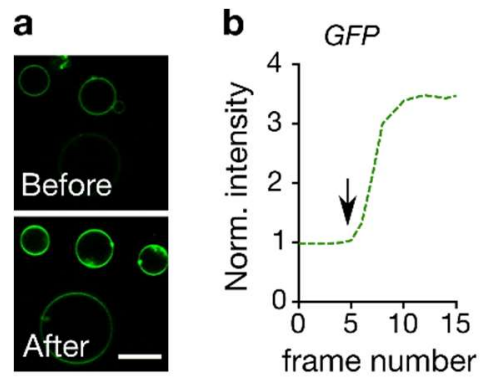
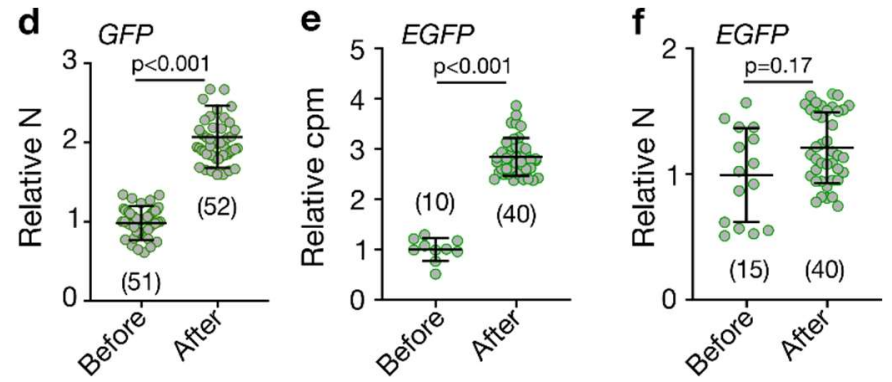

Figure 2. Effect of GFP-nanobody binding on GUV-anchored (E)GFP. Data for His-tagged (E)GFP anchored to GUVs (98 mol\% DOPC and 2 mol\% DGS-NTA) before and after addition of unlabelled $\mathrm{Nb}$ as marked. a) Representative confocal fluorescence microscopy images of the equatorial GUV plane for GFP. Scale bar $10 \mu \mathrm{m}$. b) Representative time-lapse over subsequently recorded confocal image frames of the normalized fluorescence intensity of the equatorial plane of a GFP-tagged GUV with time point of $\mathrm{Nb}$ 
171

172

173

174

175

176

177

178

179

180

181

182

183

184

185

186

187

188

189

190

191

192

193

194

195

number of particles (N) of GFP before and after $\mathrm{Nb}$ addition as marked. Relative change in (e) cpm and (d) $\mathrm{N}$ of EGFP before and after $\mathrm{Nb}$ addition as marked. Values were determined from FCS experiments on individual (E)GFP-tagged GUVs. p-values were determined using the Kolmogorov-Smirnov nonparametric test. Number of data points is indicated on each graph.

\section{Nanobody binding on live-cell membranes}

To test the effect of $\mathrm{Nb}$ on (E)GFP-tagged membrane protein organization further and in a more physiological setting, we next investigated the influence of Nbs on an GFP- and an EGFP-labelled glycosylphosphatidylinositol (GPI) anchored protein (GPI-AP) in the plasma membrane of living cells. Specifically, we expressed GFP-LYPD6 and GPI-EGFP in live PtK2 cells. GFP-LYPD6 is involved in Wnt signalling (Özhan et al., 2013), while GPI-EGFP is simply a lipid-anchored EGFP construct commonly used as probe to study GPI-AP organisation (Baumgart et al., 2016; Goswami et al., 2008; Saha et al., 2015; Schneider et al., 2017). We recorded confocal images (Figure 3a,b) as well as FCS data (Supplementary Figure 1c,d) to determine the total fluorescence intensity and values of cpm and N. For both proteins we found a modest increase in total fluorescence signal intensity upon $\mathrm{Nb}$ binding $(\approx 1.1$-fold for GFP and $\approx 1.5$-fold for EGFP, Figure $3 \mathrm{c}, \mathrm{d})$, a slight increase in molecular fluorescence brightness $(\mathrm{cpm}, \approx 1$.1-fold for GFP and $\approx 1.5$-fold for EGFP, Figure 3e,f), and a distinct variation in average number $\mathrm{N}$ of fluorescent molecules in the observation spot, with a slight $\approx 1.1$-fold increase for GFP and a slight $\approx 1$.2-fold decrease for EGFP (Figure 3c,f). However, especially the determination of the number of particles, N, was not straightforward on the live-cell membrane due to noise, cellular movements and spatial heterogeneity across the cells (i.e. due to variations in local concentrations as becomes obvious by variations in total intensity across one cell or between different cells, Figure $3 \mathrm{a}, \mathrm{b}$ ). Overall, the impact of $\mathrm{Nb}$ binding on fluorescent protein tagged GPI-AP on living cells follows the trend from the model membranes with attenuated magnitude. 

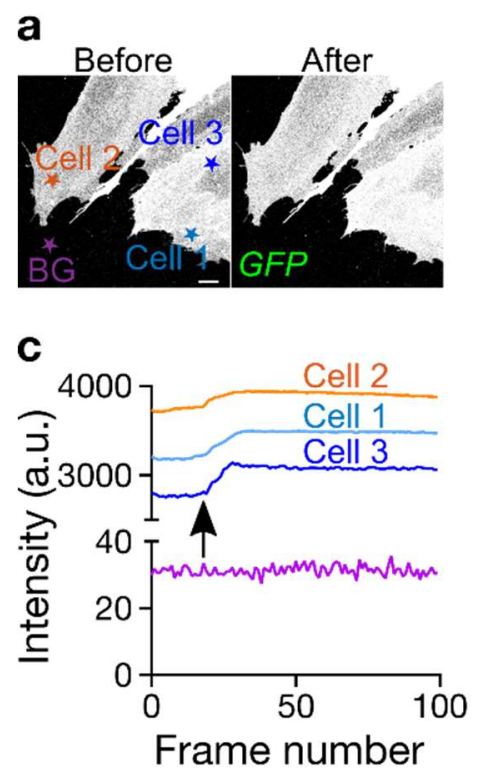

e

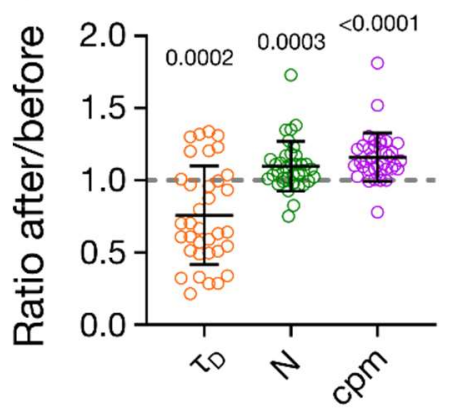

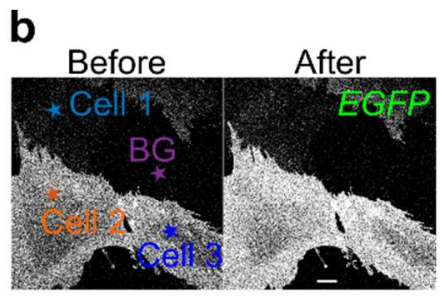

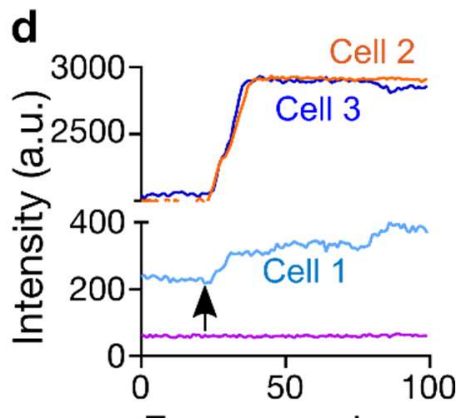

Frame number

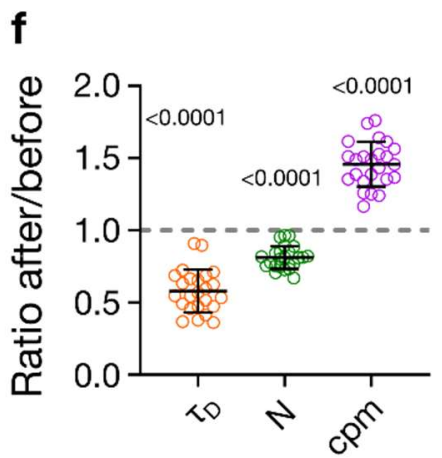

Figure 3: Effect of Nb-binding on (E)GFP in the plasma membrane of live PtK2 cells: GPI-anchored before and after addition of Nb for a) GFP-LYPD6 and b) GPI-EGFP. Scale bars are $10 \mu \mathrm{m}$. Normalised fluorescence intensity traces for c) GFP-LYPD6 and d) GPI-EGFP for the cells as indicated in panel a and $\mathrm{b}$, respectively ( $\mathrm{BG}=$ background). Arrows show the time point when $\mathrm{Nb}$ was added. The intensities per frame represent mean values over each cell (see Materials and Methods for details). Change in $\tau_{\mathrm{D}}, \mathrm{N}$ and cpm for e) GFP-LYPD6 and f) GPI-EGFP upon nanobody addition (values after Nb addition divided by values before). Change in average transit time ( $\tau_{\mathrm{D}}$ i.e. mobility), average fluorescing particle number (i.e. concentration, $\mathrm{N}$ ), and molecular fluorescence brightness (cpm) upon $\mathrm{Nb}$ addition are determined from FCS experiments (one dot $=$ one cell, for each cell $6-9$ single FCS measurements were averaged, pooled data from three different days). The values on top of ratios in e, f indicate p-values obtained from Wilcoxon sign$\mathrm{Nb}$ addition). 
212 So far, we obtained interesting insights from the stationary thermodynamic information from

213 imaging and FCS (intensity, cpm and N), but the FCS measurements also allowed us to determine

214 the average mobility of the membrane-anchored proteins. Measuring the diffusion dynamics can

215 be performed robustly against local variations in concentration and expression levels and

216 represents a way to study the organisation of plasma membrane constituents (Pinkwart et al., 2019;

217 Schneider et al., 2020). From FCS, we obtained the transit time $\tau_{\mathrm{D}}$, representing the average time

218 it takes a molecule to cross the observation spot, and tested whether it changes upon $\mathrm{Nb}$ addition

219 (Supplementary Figure 1). Intuitively, one may expect a slight decrease in mobility i.e. increase in

220 values of $\tau_{\mathrm{D}}$ upon addition of $\mathrm{Nb}$ due to the increased mass of the complex. Alternatively, one

221 could expect no change at all as the mobility of membrane constituents is overwhelmingly

222 determined by the properties of the membrane anchor (Saffman and Delbrück, 1975; Weiß et al.,

223 2013). However, interestingly, we observed an increase in mobility after $\mathrm{Nb}$ addition for GFP-

224 LYPD6 and GPI-EGFP in the membrane of live PtK2 cells (approximately 1.25 and 1.7-fold

225 decrease in values of $\tau_{\mathrm{D}}$ for GFP and EGFP, respectively; Figure $\left.3 \mathrm{e}, \mathrm{f}\right)$. From these $\tau_{\mathrm{D}}$-values and

226 the diameter $\mathrm{d}=240 \mathrm{~nm}$ of the observation spot (full-width-at-half-maximum), we can estimate

227 values of the diffusion coefficients given the diffusion equation $\left(D=\frac{d^{2}}{\ln (2) \cdot 8 \cdot \tau_{D}}\right)$ to $\mathrm{D}=0.3 \mu \mathrm{m}^{2} / \mathrm{s}$

228 and $0.4 \mu \mathrm{m}^{2} / \mathrm{s}$ for GFP-LYPD6 without and with $\mathrm{Nb}$ and $\mathrm{D}=0.8 \mu \mathrm{m}^{2} / \mathrm{s}$ and $1.4 \mu \mathrm{m}^{2} / \mathrm{s}$ for GPI-

229 EGFP without and with Nb. Previously reported values for GPI-AP diffusion scatter from 0.3 to

$2301.0 \mu \mathrm{m}^{2} / \mathrm{s}$ (Chojnacki et al., 2017; Eggeling et al., 2009; Huang et al., 2015; Lenne et al., 2006;

231 Schneider et al., 2017; Veerapathiran and Wohland, 2017) where GPI-(E)GFP typically shows

232 faster diffusion than other GPI-anchored probes (such as GPI-ACP or GPI-SNAP).

233 The apparent speed-up upon nanobody binding was puzzling, and to confirm these contradictory

234 findings, we additionally recorded FCS data of GFP-LYPD6 and GPI-EGFP in living cells with

235 higher statistical accuracy. Specifically, we performed scanning-FCS (sFCS) measurements,

236 which yield simultaneous FCS data for multiple points along a quickly scanned line, i.e. hundreds

237 of values of, for example, cpm and $\tau_{\mathrm{D}}$ within a few measurement, which accounts for spatial 238 heterogeneity and allows for the determination of average values with very high precision

239 (Schneider et al., 2018; Waithe et al., 2018). The sFCS measurements confirmed the changes in 240 values of $\tau_{\mathrm{D}}$ i.e. faster diffusion for both GFP-LYPD6 and GPI-EGFP upon Nb binding (Figure 
241 4a,b) in line with the point FCS measurements (Figure 3 e,f). Further, our sFCS data revealed that

242 the increase in mobility (i.e. decrease in transit time $\tau_{\mathrm{D}}$ ) was clearly correlated with an increase in

243 brightness, cpm (Figure 4c-f), i.e. upon $\mathrm{Nb}$ addition the population of (E)GFP tagged GPI proteins

244 shifted from a less bright and less mobile to a brighter and more mobile form.

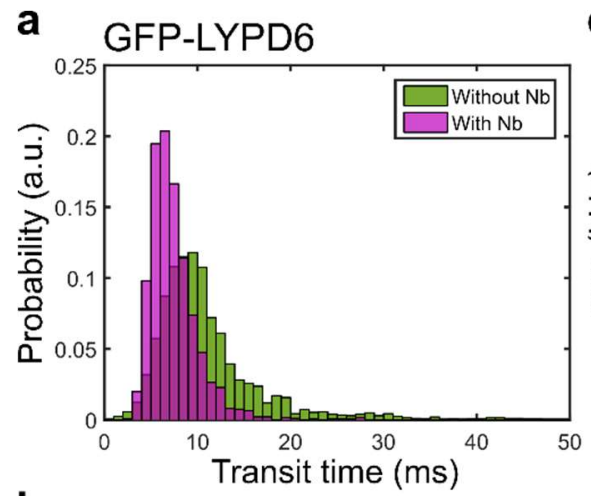

b

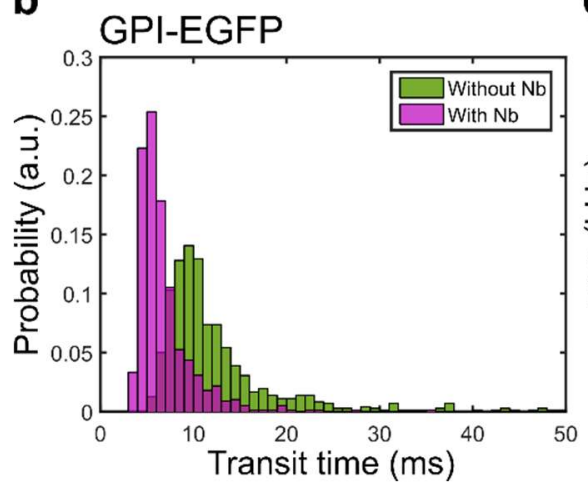

C Without $\mathrm{Nb}$

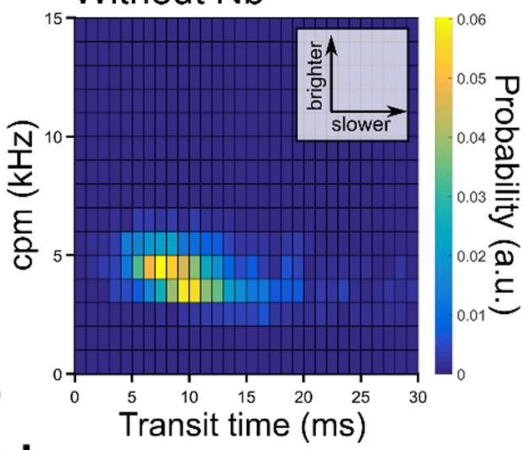

d

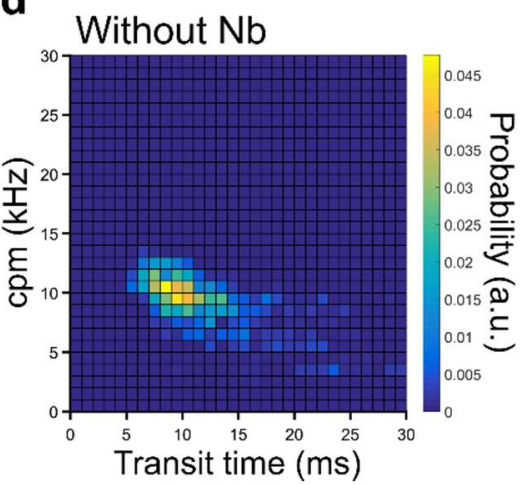

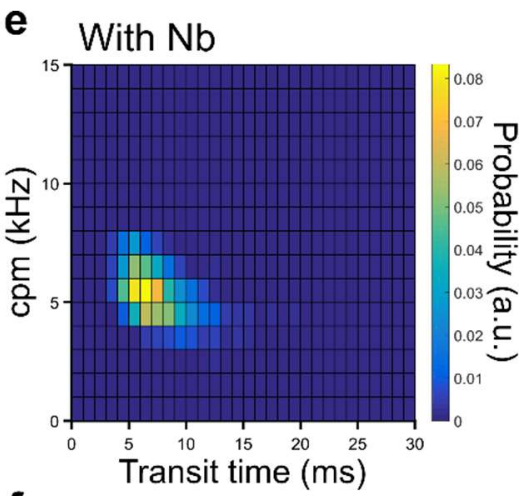

f

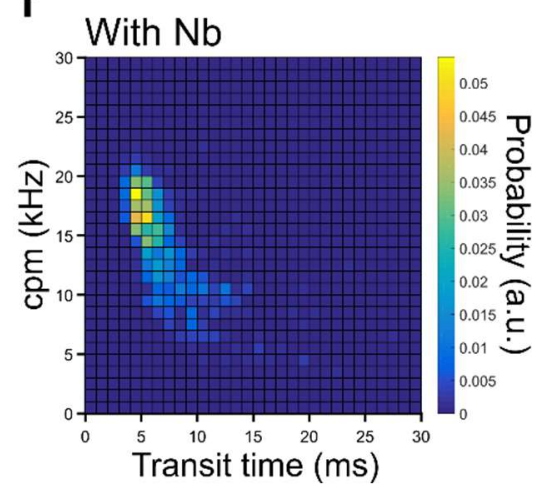

245

246

247

248

249

250

251

252

253

254

255

256

257

258

Figure 4. Effect of unlabelled Nb binding on mobility and brightness of GFP-LYPD6 and GPI-EGFP

as probed by large sFCS datasets. Analysis of diffusion dynamics and molecular brightness (cpm) of PtK2 cells expressing GFP-LYPD6 (upper panels) and GPI-EGFP (bottom panels) and the effect of unlabelled nanobody. a,b) Transit time histograms for protein without (green) and with (magenta) presence of nanobody. $>2000$ curves single FCS curves for GFP-LYPD6 and $>700$ for GPI-EGFP from $>10$ cells each. c-d) Two-dimensional pair value histograms (bi-variate histograms) of transit times and cpms for control (without Nb, c,d) and with addition of nanobody (e,f) for GFP-LYPD6 (top panels) and GPI-EGFP (bottom panels).

An explanation for these observations could be that the (E)GFP tagged proteins appear to a certain extend in aggregates or homo-oligomers that are (partially) disassembled after $\mathrm{Nb}$ binding, leading to an average increase in fluorescent particle number (Figures 2 and 3) and mobility (Figure 3 and 4) without majorly affecting the fluorescence lifetime (Supplementary Figure S2). GPI-EGFP dimers have been reported previously (Huang et al., 2015; Suzuki et al., 2012). However, since 
aggregates should in principle be brighter, one would in this case expect a decrease in molecular fluorescence brightness (cpm) upon aggregate disassembly. Our opposite observation indicates that the aggregates might actually be darker, e.g. due to self-quenching processes, and their fraction is rather low after disassembly. This is illustrated by the bi-variate histograms of cpm and $\tau_{\mathrm{D}}$ (Figure 4c-f); the fraction of darker and slower molecules ("tail" of the distribution in Figure 4c,d) is notably reduced in the presence of nanobody (Figure 4e,f). If this is indeed the case, it is essential to know whether $\mathrm{Nb}$ binds to both oligo- and monomers with different affinity or selectively to one class.

\section{Diffusion of labelled nanobodies}

To address what species is bound by the nanobody, we compared sFCS and FCS data of fluorescently labelled $\mathrm{Nb}$ and GFP-LYPD6 or GPI-EGFP at the plasma membrane of live PtK2 cells. Specifically, we employed Nbs tagged with the red-emitting dye Abberior Star 635P (AbStar635P-Nb), whose fluorescence emission was clearly distinguishable from that of the fluorescent proteins and detected on a separate detector. First, using confocal imaging we confirmed that the AbStar635-Nb bound only to the surface transfected cells and not to those without e.g. GPI-EGFP, i.e. AbStar635-Nb specifically interacted with the fluorescent protein on the membrane only (Supplementary Figure S3). Second, we also found an increase in mobility (i.e. decrease in average transit time $\tau_{\mathrm{D}}$ ) and increase in brightness cpm of the EGFP tagged proteins upon AbStar635-Nb binding, i.e. the label did not influence this effect (Figure 5a,b and Supplementary Figure S4). Interestingly, simultaneously recorded sFCS data for AbStar635P-Nb and GPI-EGFP (Figure 5c,d) revealed a profoundly slower diffusion for AbStar635P-Nb compared to the EGFP-tagged proteins. We observed an average transit time of $\tau_{\mathrm{D}}=28.3 \mathrm{~ms} \pm 1.0(\mathrm{D}=0.5$ $\left.\pm 0.02 \mu \mathrm{m}^{2} / \mathrm{s}\right)$ for AbStar635P-Nb and $\tau_{\mathrm{D}}=10.3 \mathrm{~ms} \pm 1.0\left(\mathrm{D}=1.2 \pm 0.12 \mu \mathrm{m}^{2} / \mathrm{s}\right)$ for GPI-EGFP (Figure 5a-d). This is an obvious contradiction, as the Nbs should be bound directly to the surface proteins (GPI-EGFP) but moved significantly slower than the protein itself. A possible explanation for this contradiction extends our previous hypothesis and points to the existence of two pools of (E)GFP on the cell surface; a darker oligomeric form that diffuses slowly and to which the $\mathrm{Nb}$ preferentially binds, which would drive the partial displacement of brighter and faster moving monomers, to which $\mathrm{Nb}$ does not bind efficiently. 
a
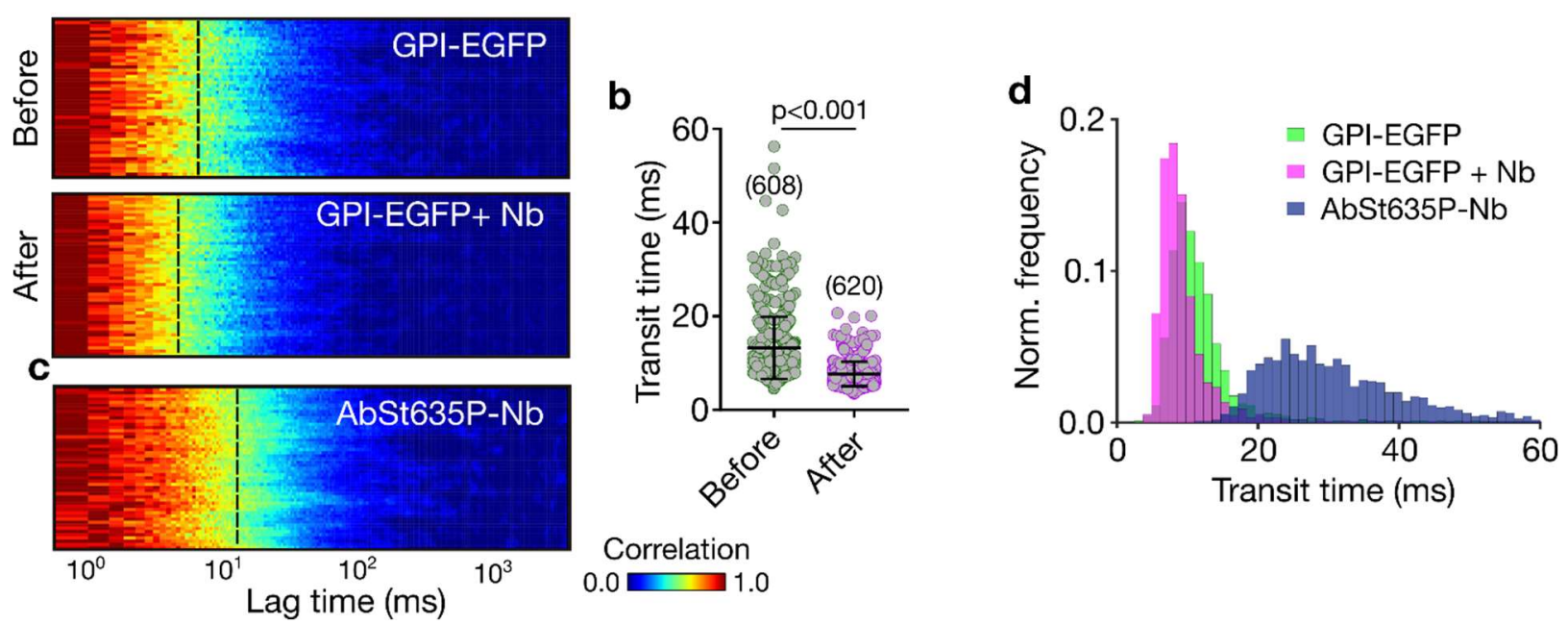

Figure 5 Diffusion of labelled Nb (AbStar635P-Nb) on GPI-EGFP expressing PtK2 cells. a,b)

Representative normalised sFCS autocorrelation carpets for (a) GPI-EGFP before and after addition of AbStar635P-Nb (x: correlation lag time, y: line pixels (space), colour scale: normalised correlation from zero (blue) to one (red)), revealing a shift of the decay of the correlation curves (yellow region, average transit time highlighted by the dashed line) towards shorter times after addition of labelled $\mathrm{Nb} . \mathrm{b}) \tau_{\mathrm{D}}$ values for GPI-EGFP before and after AbStar635P-Nb addition including mean values and standard deviations. c) Normalised autocorrelation carpet for AbStar635P-Nb bound to PTK2 cells expressing GPI-EGFP. d) Histogram of $\tau_{\mathrm{D}}$ for GPI-EGFP (with and without $\mathrm{Nb}$ ) and AbStar635P-Nb. The p-value given in panel $b$ was calculated using the Kolmogorov-Smirnov non-parametric test.

To investigate these indications further, we applied fluorescence cross correlation spectroscopy (FCCS (Schwille et al., 1997)). Based on the principle of FCS, FCCS takes information from the temporal cross-correlation function of two simultaneously recorded fluorescence signal time traces of two distinctively labelled and emitting (e.g. green and red fluorescence, respectively) diffusing molecules to determine the degree of co-diffusion or interaction of the two molecules. Only when molecules show co-diffusion or interaction, the amplitude of the cross-correlation curve is larger than zero. An FCCS amplitude of zero indicates the absence of co-diffusion of the two fluorescing molecules (Bacia and Schwille, 2007). Therefore, the AbStar635P-Nb binding to fluorescently tagged GPI-APs should be a perfect sample for FCCS analysis, since every (red-emitting) $\mathrm{Nb}$ molecule should be bound to a (green-emitting) (E)GFP, yielding in theory a perfect crosscorrelation between the red and green fluorescence signals. Performance of our FCCS experiments 


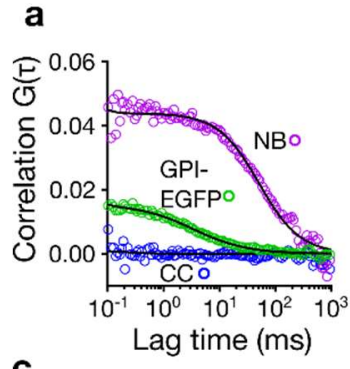

C

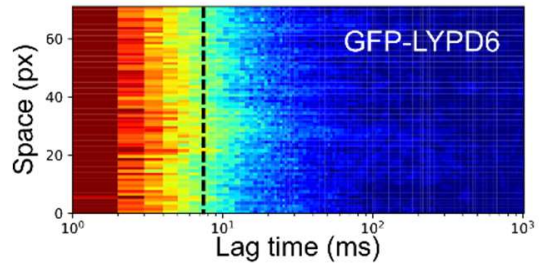
diffusing entity (supposedly oligomers). b

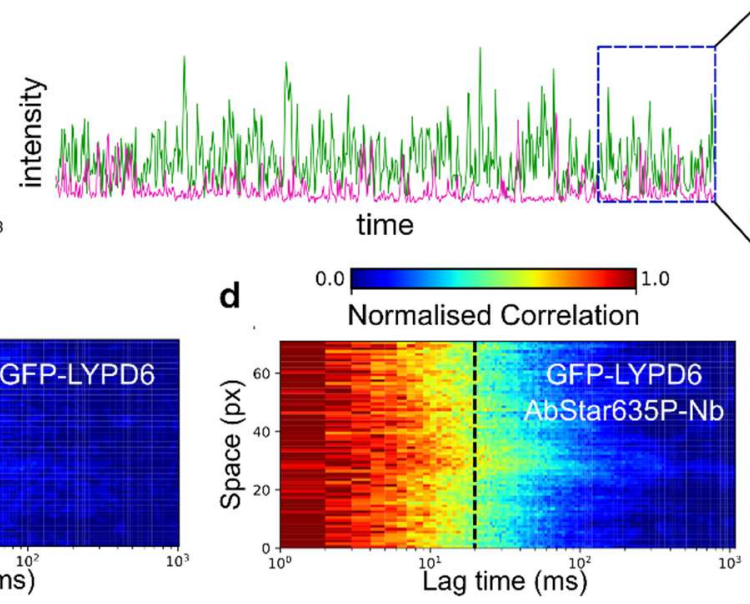
(E)GFPtagged molecules anchored to the plasma membrane, as shown for GPI-EGFP in Figure 6a. The absence of co-diffusion is also illustrated by the dual-colour intensity time trace (Figure 6b) demonstrating only very rare detection events with signal from both channels, i.e. EGFP and AbStar635P-Nb independently crossed the observation spot. Following the same strategy as before, we used sFCS to confirm these findings for GFP-LYPD6, employing higher statistical throughput and spatial sampling. The auto-correlation carpets (Figure 6c,d) reveal the same slower diffusion of the bound AbStar635P-Nb compared to the binding partner GFP-LYPD6, as was the case for the experiments with GPI-EGFP (Figure 5 a,c). Similarly the scanning cross-correlation data of AbStar635P-Nb and GFP-LYPD6 confirmed the complete absence of co-diffusion of fluorescing binding partners and in contrast to a positive control (Supplementary Figure S6), we only observed noise (Figure 6e). We can only conclude that the Nbs do not bind the bright and fast diffusing (E)GFP-tagged proteins (supposedly monomers), but predominantly to a dark and slowly

Figure 6 Missing co-diffusion of labelled $\mathrm{Nb}$ and (E)GFP-tagged surface proteins. PtK2 cells expressing GPI-EGFP or GFP-LYPD6 were treated with labelled Nb (AbStar635P-Nb) and FCCS data acquired. Positive cross correlation (CC) indicates interaction, i.e. co-diffusion. a) point FCCS of GPIEGFP with autocorrelation EGFP (green), autocorrelation Nb (labelled with AbberiorStar635P, magenta) 
and CC (blue). b) Representative dual-colour intensity trace showing that the detection events for EGFP and AbStar635P-Nb rarely overlap in time. c-e) scanning FCCS data of GFP-LYPD6 expressed on the surface of PtK2 cells, with representative normalised auto-correlation data for (c) GFP-LYPD6, (d) AbStar635P-Nb and (e) normalised cross-correlation data of those. The dashed black line indicates the average transit times. The temporal cross-correlation of these two dataset does not show any positive crosscorrelation, i.e. no co-diffusion.

Other reasons for the absence of cross-correlation could be i) very fast binding kinetics (i.e. onand off-rates) of the $\mathrm{Nb}-(\mathrm{E}) \mathrm{GFP}$ interaction or $\mathrm{ii}$ ) too low binding leaving too many unbound (E)GFP molecules. However, our data do not support these scenarios. 1) We determined off-rates, $\mathrm{k}_{\mathrm{off}}$, for the $\mathrm{Nb}$-GFP binding using surface plasmon resonance (SPR) experiments (of GFP binding to surface-immobilised (labelled and unlabelled) $\mathrm{Nbs}$ ), resulting in $\mathrm{k}_{\text {off }} \approx 5 \times 10^{-4} \mathrm{~s}^{-1}$ (Supplementary Figure S7). Consequently, the Nb-(E)GFP complex is stable for about 30 minutes, which is in agreement with previous data (Della Pia and Martinez, 2015). During the 1-50 milliseconds long transit through the observation spot, the Nb-(E)GFP complex should be intact. This data rules out fast kinetics. 2) We also recorded FCCS data in large excess of AbStar635-Nb, saturating GFP binding. However, we still did not observe any cross-correlation (Supplementary Figure S8). This data rules out the domination of unbound particles.

We also tested whether the lack of cross-correlation signal could be dye-specific for AbStar635P, but cross-correlation was also absent when performing the same sFCCS experiments as before with nanobody labelled with the dye Atto594 (Supplementary Figure S9).

Another possible explanation for the lack of co-diffusion might be an extremely efficient (close to 100\%) energy transfer (Sun et al., 2011) between the fluorescent protein(s) and the AbStar635$\mathrm{Nb}$. Such a Förster resonance energy transfer (FRET) would render the Nb-bound fluorescent proteins very dim and therefore hardly visible for FCCS analysis (FCS-based experiments require rather large fluorescence brightness (Saffarian and Elson, 2003; Schneider et al., 2020)). Several observations oppose this scenario: i) Close to 100\% FRET efficiency should lead to a huge decrease in the number $\mathrm{N}$ of visible donor (E)GFP molecules and thus a decrease of EGFP fluorescence intensity with nanobody binding, which is not the case (Figure 1, 2 and Supplementary Figure S10). ii) A large energy transfer generally leads to a vast decrease in the 
fluorescence lifetime of the FRET donor, in this case (E)GFP. We therefore measured and compared values of the fluorescence lifetime for GFP and EGFP with and without binding to unlabelled and AbStar635P-labelled $\mathrm{Nb}$. There was a small reduction in fluorescence lifetime for GFP $(\approx 3 \mathrm{~ns}$ to $\approx 2.2 \mathrm{~ns})$ and $\mathrm{EFGP}(\approx 2.6 \mathrm{~ns}$ to $\approx 2.1 \mathrm{~ns})$ in solution (Supplementary Figure S11), indicating only a minor influence by FRET and not explaining the complete lack of crosscorrelating fluorescence signal. Some FRET may explain though the lower increase in fluorescence intensity upon labelled nanobody binding to the recombinant proteins in solution (Supplementary Figure 12) compared to unlabelled Nb (Figure 1). iii) The molecular fluorescence brightness cpm of the donor EGFP molecules should go down after addition of labelled compared to unlabelled Nbs. As highlighted before, we however see an increase in cpm values independent of labelled or unlabelled Nb (Figure 4c-f and Supplementary Figure S4).

\section{Conclusions}

The use of Nbs, not only as an alternative for full-length antibodies, presents a versatile new route for detection and manipulation of proteins in biology and especially microscopy (Beghein and Gettemans, 2017). Their small size, monovalancy, the ability to label them stoichiometrically, and their recombinant or even in vivo production makes them an attractive tool (Bothma et al., 2018; Grußmayer et al., 2014; Sograte-Idrissi et al., 2020). The GFP-binding Nbs along with Nbs against other fluorescent proteins enable conveniently to perform super-resolution microscopy on the tagged protein of interest (Platonova et al., 2015; Ries et al., 2012; Sograte-Idrissi et al., 2019). A modulation of the GFP's spectral properties by interaction with Nbs has been shown and exploited before (Bothma et al., 2018; Kirchhofer et al., 2010), however the Nb has not been implicated in the reorganisation of the tagged protein.

In this study, we used imaging and spectroscopic techniques to investigate changes in the dynamic organisation of (E)GFP tagged lipid anchors in model membranes and living cells upon binding of labelled and unlabelled anti-GFP nanobody. Overall, our data show that (i) $\mathrm{Nb}$ increases the apparent number of GFP molecules on GUVs and cells, (ii) Nb binding to fluorescent proteins on living cells increases the mobility of GPI-anchored EGFP or GFP, (iii) the Nb diffuses slowly compared to the GFP on living cells, which means that they do not co-diffuse, and (iv) the $\mathrm{Nb}$ binding modulates photophysical properties of the fluorescent proteins. These findings may suggest that the Nbs bind predominantly to an already existing dark form of the (E)GFP, which 
might be a slow-moving, higher order complex. Lack of co-diffusion suggests that this complex is not strongly fluorescent (e.g., due to self-quenching) but still primarily recognized by the $\mathrm{Nb}$. Upon binding, $\mathrm{Nb}$ could partially disassemble the dark complex and release a few molecules from the complex that become fluorescent but are not bound to a $\mathrm{Nb}$. Although this process still needs to be directly shown in the future, our results imply that the $\mathrm{Nb}$ binding could influence the organization of the GFP-tagged proteins in living cells. It has been reported before that $\mathrm{Nb}$ binding could perturb protein function (Küey et al., 2019). Consequently, when performing conventional or super-resolved imaging using anti-GFP nanobodies to label (E)GFP conjugated proteins, the measurements need to be interpreted with care, especially on live cells and when quantitative data on dynamics are derived.

We only showed the effect of GFP-nanobodies, thus we refrain ourselves from generalizing the effect to all nanobodies. Recently, by using SNAP-25 and Syntaxin 1A nanobodies, a previously undetected pool of synaptic population were found in the cells (Maidorn et al., 2019), which was attributed to nanobodies' ability to reveal different organization patterns. Therefore, there is a possibility that the effect of nanobodies could be more general. Moreover, here we only test nanobodies against GFP proteins (in model membranes) or GFP-labelled GPI-anchored proteins, but it seems probable that the effect will be similar on other proteins labelled with GFP or its derivatives as the modulating interactions are between the fluorescent protein and the nanobody.

\section{Significance}

Nanobodies, especially against fluorescent proteins, are widely used in microscopy to investigate the organisation of recombinantly tagged proteins. Usually, the fluorescent label on the nanobody is imaged as proxy for the organisation of the protein of interest. Here, by applying imaging and single molecule fluorescence spectroscopy, we show that in live cells, the distribution and dynamics of nanobody and target protein of interest may differ. We used GPI-anchored proteins as an example and illustrate that the nanobody bound to GPI-EGFP did not accurately resemble the native organisation of the protein and may even change it. Since nanobodies are constantly becoming more popular, our findings are crucial as they suggest that it is necessary to exercise prudence when applying nanobodies for quantitative analysis of live-cell microscopy.

\section{Acknowledgements}


421 We thank the EMBL Super-resolution course, Dr. Marco Lampe and Dr. Jonas Ries for their help 422 on emergence of this interesting question, Dr. Chris Paluch for his help on Biacore SPR analysis.

423 We also thank Christoffer Lagerholm and the Wolfson Imaging Centre Oxford and the Micron 424 Advanced Bioimaging Unit (Wellcome Trust Strategic Award 091911) for providing microscope 425 facility and financial support. We acknowledge funding by the Wolfson Foundation, the Medical 426 Research Council (MRC, grant number MC_UU 12010/unit programmes G0902418 and 427 MC_UU_12025), MRC/BBSRC/EPSRC (grant number MR/K01577X/1), the Wellcome Trust 428 (grant ref 104924/14/Z/14), the Deutsche Forschungsgemeinschaft (Research unit 1905 "Structure 429 and function of the peroxisomal translocon", Jena Excellence Cluster "Balance of the Microverse", 430 Collaborative Research Center 1278 "Polytarget"), Oxford-internal funds (John Fell Fund and 431 EPA Cephalosporin Fund) and Wellcome Institutional Strategic Support Fund (ISSF). ES is 432 funded by the Newton-Katip Celebi Institutional Links grant (352333122) and SciLifeLab 433 fellowship.

Author contributions

435 F.S. and E.S. designed the study, performed the experiments and analysed the data. F.S., E.S. and 436 C.E. wrote the manuscript.

437 Declaration of interest

438 The authors declare no conflict of interest

439 Materials and Methods

$440 \quad$ Cell culture \& labelling

441 PtK2 cells were cultured at $37{ }^{\circ} \mathrm{C}, 5 \% \mathrm{CO}_{2}$, in DMEM (Sigma Aldrich) supplemented with 16\% 442 FBS (Sigma Aldrich). For microscopy the cells were seeded on $25 \mathrm{~mm}$ diameter glass coverslips 443 (\#1.5 thickness). Transfections of GPI-EGFP (kind gift from Kai Simon's lab) and GFP-LYPD6 444 (Özhan et al., 2013) were performed with Lipofectamine 3000 (Thermo Fisher) according to the 445 manufacturer's protocol.

446 While imaging cells at $37{ }^{\circ} \mathrm{C}$, they were incubated with unlabelled (GFP-binding protein, 447 Chromotek) and Abberior 635P conjugated Nanobody (GFP-booster, Chromotek). All 
448

449

450

451

452

453

454

455

456

457

458

459

460

461

462

463

464

465

466

467

468

469

470

471

472

473

474

experiments were performed in L15 imaging medium (Sigma Aldrich) and the nanobody added to $1 \mathrm{~mL}$ and well mixed.

GUVS

GUVs were prepared using electro-formation as described in(Jenkins et al., 2019). Lipid mixture (1mg/mL DOPC:DGS-Ni-NTA (both obtained from Avanti Polar Lipids) 96:4 molar ratio) were spread on platinum wire and dipped into $300 \mathrm{mM}$ sucrose. GUVs formed after exposure to an AC filed of $2 \mathrm{~V}$ and $10 \mathrm{~Hz}$ for $1 \mathrm{~h}$ followed by $2 \mathrm{~V} 2 \mathrm{~Hz}$ for another 30 minutes. His-tagged GFP (Sino Biological) and eGFP (OriGene) was incubated for 20 minutes with the vesicles before imaging and FCS was performed.

\section{Confocal microscopy \& FCS}

Confocal microscopy and FCS were performed on a Zeiss 780 and Zeiss 880 both equipped with an Argon laser for fluorescence excitation. All sFCS and most imaging has been performed in photon counting mode using Channel S. To excite the labelled nanobody the HeNe 633 excitation has been employed. For single colour FCS and imaging a 488 dichroic mirror and for two-colour imaging a 488/561/633 MBS was used. The fluorescence was collected between $500 \mathrm{~nm}$ and 600 $\mathrm{nm}$ for the green channel and between $640 \mathrm{~nm}$ and $695 \mathrm{~nm}$ for the red channel. Laser powers were between 1 and $5 \mu \mathrm{W}$ and kept below saturation to avoid artefacts in FCS.

The images were processed using FIJI (Rueden et al., 2017; Schindelin et al., 2012). The plasma membranes of each cell was segmented out using the polygon selection tool. Similar sized regions of interest were generated for the background. The average intensities over time for the whole area were extracted from the z-profile.

Point-FCS measurements were performed using Zeiss' internal FCS routine. Measurements were between 10 and 15 seconds long. The objective's correction collar was adjusted and the pinhole aligned measuring the diffusion of Alexa Fluor 488 in water. FCS measurements were saved as .fcs files for fitting. The same procedure was followed for cross-correlation (FCCS) measurements but additionally using a cross-correlation positive control (Bodipy and Alexa647 labelled HDL particles (Plochberger et al., 2017)) to ensure optical alignment. 
sFCS measurements were performed as xt scans. 52 pixels were acquired for $10^{5}$ lines at about $2000 \mathrm{~Hz}$ yielding pixel dwell time of $3.94 \mu$ s (overall resulting in a total acquisition time of about 47 seconds). The data were saved as .lsm5 file and externally correlated using the FoCuS_scan software package (Waithe et al., 2018). To correct for photobleaching, the first seconds were cropped off and a local averaging bleaching-correction applied as described in (Waithe et al., 2018). sFCCS measurements were performed in a similar manner using the described acquisition settings in conjunction with the optical set-up for two-colour imaging as described above. As a positive control for sFCCS, we used a sparse sample of DOPC (1,2-dioleoyl-sn-glycero-3phosphocholine), Avanti Polar Lipids) vesicles doped 1:50,000 with DiO (Invitrogen) and 1:10,000 with AbberiorSTAR-Red-PEG-Cholesterol (Abberior). The sample was prepared by mixing the lipid and dyes in ethanol, drying the mixture, and re-suspending the lipid film by vortexing and ultra-sonication (5 minutes and 30 minutes, respectively) in water. Measurements were performed in PBS.

Point and sFCS data were fitted in FoCuS (Waithe et al., 2016, 2018). Point-FCS data showed a contribution of a triplet component (40 $\mu$ s for (E)GFP (Schneider et al., 2020)), a fast (probably cytoplasmic) component, with transit times around $0.1 \mathrm{~ms}$, and a slower transit time which was attributed to the diffusion in the membrane. Thus, pFCS data were fitted with a two component diffusion model including a triplet state (Widengren et al., 1995). sFCS acquisitions miss the fast dynamics and were fitted with a single component 2D diffusion model (Schneider et al., 2018). Fitting in $\mathrm{FoCuS}$ is performed using a Levenberg-Marquard non-linear least suare optimisation. The fitted parameters including the cpms (determined from fitted amplitude and the average count rate) were exported as Excel sheets and post processed with Matlab (Schneider et al., 2018).

Some data on mobility were acquired cell by cell to account for the inherent biological heterogeneity. In these cases only the ratio of the transit times, number of molecules or counts per molecule before and after addition of the nanobody are reported (After/Before). Statistical tests were performed in GraphPad Prism 8. We employed the Wilcoxon sign-rank non-parametric tests with hypothetical median values of 1 for the data presented as ratios and we used the KolmogorovSmirnov non-parametric test for all other data.

\section{Lifetime measurements}


504 Life time imaging was performed on a Microtime 200 (PicoQuant) equipped with a FlimBee galvo

505 scanner. Fluorescence was excited with a $488 \mathrm{~nm}$ diode laser (PicoQuant) and focused onto the 506 sample with an Olympus UPlanSApo $60 \mathrm{x}$ water-immersion objective. Images were acquired as

50750 by $50 \mu^{2}$ (512 by 512 pixel) for collecting the fluorescence for $60 \mathrm{~s}$ at low excitation power

$508(<1 \mu \mathrm{W})$ to avoid too high count rates and distortion of the TCSPC data (Isbaner et al., 2016). The

509 overall TCSPC curves were used for lifetime fitting (2 component tail fit, second component fixed

510 to $4.1 \mathrm{~ns})$. We report the amplitude weighted lifetime of GPI-eGFP and GFP-LYPD6 in the plasma

511 membrane of living PtK2 cells.

512 The same set-up was used to measure the fluorescent lifetime of GFP-His and EGFP-His in

513 solution. The data were acquired as point scans.

$514 S P R$

515 We immobilised the nanobodies (either fluorescently labelled or unlabelled) by amine coupling to 516 a CM5 chip (with an RFPNB in the reference channel) then injected GFP as the analyte in a kinetic 517 analysis (a single injection at $87 \mathrm{nM}$, using curve fitting in the BiaEvaluation software to measure 518 on and off rates from which the $\mathrm{K}_{\mathrm{D}}$ is calculated).

\section{Spectra}

520 All spectral measurements were performed using a CLARIO STAR plate reader (BMG

521 LABTECH). His-tagged GFP and EGFP were measured in PBS in glass bottom 96-well plates

522 (Porvair Sciences) which were prior to the measurements coated with BSA to prevent sticking of 523 the fluorescent proteins to the glass. All spectra are averages of multiple wells. Excitation scan 524 were performed with a readout at $510 \mathrm{~nm}$ and emission scans were performed with excitation at $525405 \mathrm{~nm}$ or $488 \mathrm{~nm}$. We choose a spectral resolution of $1 \mathrm{~nm}$ and used 200 flashes per wavelength 526 for averaging. 


\section{References}

528

529

530

531

532

533

534

535

536

537

538

539

540

541

542

543

544

545

546

547

548

549

550

551

552

553

554

555

556

557

558

559

560

561

562

563

564

Aguilar, G., Matsuda, S., Vigano, M.A., and Affolter, M. (2019). Using Nanobodies to Study Protein Function in Developing Organisms. Antibodies 8, 16.

Arbabi Ghahroudi, M., Desmyter, A., Wyns, L., Hamers, R., and Muyldermans, S. (1997). Selection and identification of single domain antibody fragments from camel heavy-chain antibodies. FEBS Lett. 414, 521-526.

Bacia, K., and Schwille, P. (2007). Practical guidelines for dual-color fluorescence crosscorrelation spectroscopy. Nat. Protoc. 2, 2842-2856.

Baumgart, F., Arnold, A.M., Leskovar, K., Staszek, K., Fölser, M., Weghuber, J., Stockinger, H., and Schütz, G.J. (2016). Varying label density allows artifact-free analysis of membrane-protein nanoclusters. Nat. Methods 2, 0-10.

Beghein, E., and Gettemans, J. (2017). Nanobody Technology: A Versatile Toolkit for Microscopic Imaging, Protein-Protein Interaction Analysis, and Protein Function Exploration. Front. Immunol. 8, 1-14.

Bothma, J.P., Norstad, M.R., Alamos, S., and Garcia, H.G. (2018). LlamaTags: A Versatile Tool to Image Transcription Factor Dynamics in Live Embryos. Cell 173, 1810-1822.e16.

Braun, M.B., Traenkle, B., Koch, P.A., Emele, F., Weiss, F., Poetz, O., Stehle, T., and Rothbauer, U. (2016). Peptides in headlock - A novel high-affinity and versatile peptide-binding nanobody for proteomics and microscopy. Sci. Rep. 6, 1-10.

Buser, D.P., Schleicher, K.D., Prescianotto-Baschong, C., and Spiess, M. (2018). A versatile nanobody-based toolkit to analyze retrograde transport from the cell surface. Proc. Natl. Acad. Sci. U. S. A. 115, E6227-E6236.

Cao, J., Zhong, N., Wang, G., Wang, M., Zhang, B., Fu, B., Wang, Y., Zhang, T., Zhang, Y., Yang, K., et al. (2019). Nanobody-based sandwich reporter system for living cell sensing influenza A virus infection. Sci. Rep. 9, 15899.

Carrington, G., Tomlinson, D., and Peckham, M. (2019). Exploiting nanobodies and Affimers for superresolution imaging in light microscopy. Mol. Biol. Cell 30, 2737-2740.

Chattoraj, M., King, B.A., Bublitz, G.U., and Boxer, S.G. (1996). Ultra-fast excited state dynamics in green fluorescent protein: multiple states and proton transfer. Proc. Natl. Acad. Sci. 93, 8362-8367.

Chojnacki, J., Waithe, D., Carravilla, P., Huarte, N., Galiani, S., Enderlein, J., and Eggeling, C. (2017). Envelope glycoprotein mobility on HIV-1 particles depends on the virus maturation state. Nat. Commun. 8, 545.

Chudakov, D.M., and Lukyanov, K.A. (2003). Use of Green Fluorescent Protein (GFP) and Its Homologs for in vivo Protein Motility Studies. Biochem. 68, 952-957.

Conyard, J., Kondo, M., Heisler, I.A., Jones, G., Baldridge, A., Tolbert, L.M., Solntsev, K.M., and Meech, S.R. (2011). Chemically modulating the photophysics of the GFP chromophore. J. Phys. Chem. B 115, 1571-1577. 
Eggeling, C., Ringemann, C., Medda, R., Schwarzmann, G., Sandhoff, K., Polyakova, S., Belov, V.N., Hein, B., von Middendorff, C., Schönle, A., et al. (2009). Direct observation of the nanoscale dynamics of membrane lipids in a living cell. Nature 457, 1159-1162.

Fabricius, V., Lefèbre, J., Geertsema, H., Marino, S.F., and Ewers, H. (2018). Rapid and efficient C-terminal labeling of nanobodies for DNA-PAINT. J. Phys. D. Appl. Phys. 51.

Farrants, H., Tarnawski, M., Müller, T.G., Otsuka, S., Hiblot, J., Koch, B., Kueblbeck, M., Kräusslich, H.-G., Ellenberg, J., and Johnsson, K. (2020). Chemogenetic Control of Nanobodies. Nat. Methods 683557.

Ghosh, A., Isbaner, S., Veiga Gutierrez, M., Gregor, I., Enderlein, J., and Karedla, N. (2017). Quantifying Microsecond Transition Times Using Fluorescence Lifetime Correlation Spectroscopy. J. Phys. Chem. Lett. acs.jpclett.7b02707.

Goswami, D., Gowrishankar, K., Bilgrami, S., Ghosh, S., Raghupathy, R., Chadda, R., Vishwakarma, R., Rao, M., and Mayor, S. (2008). Nanoclusters of GPI-Anchored Proteins Are Formed by Cortical Actin-Driven Activity. Cell 135, 1085-1097.

Grußmayer, K.S., Kurz, A., and Herten, D.P. (2014). Single-molecule studies on the label number distribution of fluorescent markers. ChemPhysChem 15, 734-742.

Harmsen, M.M., and De Haard, H.J. (2007). Properties, production, and applications of camelid single-domain antibody fragments. Appl. Microbiol. Biotechnol. 77, 13-22.

Huang, H., Simsek, M.F., Jin, W., and Pralle, A. (2015). Effect of receptor dimerization on membrane lipid raft structure continuously quantified on single cells by camera based fluorescence correlation spectroscopy. PLoS One 10, 1-18.

Isbaner, S., Karedla, N., Ruhlandt, D., Stein, S.C., Chizhik, A., Gregor, I., and Enderlein, J. (2016). Dead-time correction of fluorescence lifetime measurements and fluorescence lifetime imaging. Opt. Express 24, 9429-9445.

Jenkins, E., Santos, A.M., O’Brien-Ball, C., Felce, J.H., Wilcock, M.J., Hatherley, D., Dustin, M.L., Davis, S.J., Eggeling, C., and Sezgin, E. (2019). Reconstitution of immune cell interactions in free-standing membranes. J. Cell Sci. 132, jcs219709.

Jullien, D., Vignard, J., Fedor, Y., Béry, N., Olichon, A., Crozatier, M., Erard, M., Cassard, H., Ducommun, B., Salles, B., et al. (2016). Chromatibody, a novel non-invasive molecular tool to explore and manipulate chromatin in living cells. J. Cell Sci. 129, 2673-2683.

Jung, G., Wiehler, J., and Zumbusch, A. (2005). The photophysics of green fluorescent protein: Influence of the key amino acids at positions 65, 203, and 222. Biophys. J. 88, 1932-1947.

Kirchhofer, A., Helma, J., Schmidthals, K., Frauer, C., Cui, S., Karcher, A., Pellis, M., Muyldermans, S., Casas-Delucchi, C.S., Cardoso, M.C., et al. (2010). Modulation of protein properties in living cells using nanobodies. Nat. Struct. Mol. Biol. 17, 133-138.

Klamecka, K., Severin, P.M., Milles, L.F., Gaub, H.E., and Leonhardt, H. (2015). Energy profile of nanobody-GFP complex under force. Phys. Biol. 12, 056009.

Küey, C., Larocque, G., Clarke, N.I., and Royle, S.J. (2019). Unintended perturbation of protein 
function using GFP nanobodies in human cells. J. Cell Sci. 132, jcs234955.

Lenne, P.-F., Wawrezinieck, L., Conchonaud, F., Wurtz, O., Boned, A., Guo, X.-J., Rigneault, H., He, H.-T., and Marguet, D. (2006). Dynamic molecular confinement in the plasma membrane by microdomains and the cytoskeleton meshwork. EMBO J. 25, 3245-3256.

Leslie, M. (2018). Small but mighty. Science (80-. ). 360, 594-597.

Lippincott-Schwartz, J., and Patterson, G.H. (2009). Photoactivatable fluorescent proteins for diffraction-limited and super-resolution imaging. Trends Cell Biol. 19, 555-565.

Maidorn, M., Olichon, A., Rizzoli, S.O., and Opazo, F. (2019). Nanobodies reveal an extrasynaptic population of SNAP-25 and Syntaxin 1A in hippocampal neurons. MAbs 11, 305-321.

Maier, J., Traenkle, B., and Rothbauer, U. (2015). Real-time analysis of epithelial-mesenchymal transition using fluorescent single-domain antibodies. Sci. Rep. 5, 1-13.

Mikhaylova, M., Cloin, B.M.C., Finan, K., Van Den Berg, R., Teeuw, J., Kijanka, M.M., Sokolowski, M., Katrukha, E.A., Maidorn, M., Opazo, F., et al. (2015). Resolving bundled microtubules using anti-tubulin nanobodies. Nat. Commun. 6.

Niwa, H., Inouye, S., Hirano, T., Matsuno, T., Kojima, S., Kubota, M., Ohashi, M., and Tsuji, F.I. (1996). Chemical nature of the light emitter of the Aequorea green fluorescent protein. Proc. Natl. Acad. Sci. U. S. A. 93, 13617-13622.

Özhan, G., Sezgin, E., Wehner, D., Pfister, A.S., Kühl, S.J., Kagermeier-Schenk, B., Kühl, M., Schwille, P., and Weidinger, G. (2013). Lypd6 Enhances Wnt/ $\beta$-Catenin Signaling by Promoting Lrp6 Phosphorylation in Raft Plasma Membrane Domains. Dev. Cell 26, 331-345.

Pereira, P.M., Albrecht, D., Culley, S., Jacobs, C., Marsh, M., Mercer, J., and Henriques, R. (2019). Fix Your Membrane Receptor Imaging: Actin Cytoskeleton and CD4 Membrane Organization Disruption by Chemical Fixation. Front. Immunol. 10, 675.

Della Pia, E.A., and Martinez, K.L. (2015). Single domain antibodies as a powerful tool for high quality surface plasmon resonance studies. PLoS One 10,1-17.

Pinkwart, K., Schneider, F., Lukoseviciute, M., Sauka-Spengler, T., Lyman, E., Eggeling, C., and Sezgin, E. (2019). Nanoscale dynamics of cholesterol in the cell membrane. J. Biol. Chem. 294, 12599-12609.

Platonova, E., Winterflood, C.M., Junemann, A., Albrecht, D., Faix, J., and Ewers, H. (2015). Single-molecule microscopy of molecules tagged with GFP or RFP derivatives in mammalian cells using nanobody binders. Methods 88, 89-97.

Pleiner, T., Bates, M., Trakhanov, S., Lee, C.T., Schliep, J.E., Chug, H., Böhning, M., Stark, H., Urlaub, H., and Görlich, D. (2015). Nanobodies: Site-specific labeling for super-resolution imaging, rapid epitope- mapping and native protein complex isolation. Elife 4, 1-21.

Pleiner, T., Bates, M., and Görlich, D. (2018). A toolbox of anti-mouse and anti-rabbit IgG secondary nanobodies. J. Cell Biol. 217, 1143-1154.

Plochberger, B., Röhrl, C., Preiner, J., Rankl, C., Brameshuber, M., Madl, J., Bittman, R., Ros, R., Sezgin, E., Eggeling, C., et al. (2017). HDL particles incorporate into lipid bilayers - a 
641

642

643

644

645

646

647

648

649

650

651

652

653

654

655

656

657

658

659

660

661

662

663

664

665

666

667

668

669

670

671

672

673

674

675

676

677

678

combined AFM and single molecule fluorescence microscopy study. Sci. Rep. 7, 15886.

Ries, J., Kaplan, C., Platonova, E., Eghlidi, H., and Ewers, H. (2012). A simple, versatile method for GFP-based super-resolution microscopy via nanobodies. Nat. Methods 9, 582-584.

Rueden, C.T., Schindelin, J., Hiner, M.C., DeZonia, B.E., Walter, A.E., Arena, E.T., and Eliceiri, K.W. (2017). ImageJ2: ImageJ for the next generation of scientific image data. BMC Bioinformatics 18, 529 .

Saffarian, S., and Elson, E.L. (2003). Statistical analysis of fluorescence correlation spectroscopy: The standard deviation and bias. Biophys. J. 84, 2030-2042.

Saffman, P.G., and Delbrück, M. (1975). Brownian motion in biological membranes. Proc. Natl. Acad. Sci. U. S. A. 72, 3111-3113.

Saha, S., Lee, I.-H., Polley, A., Groves, J.T., Rao, M., and Mayor, S. (2015). Diffusion of GPIanchored proteins is influenced by the activity of dynamic cortical actin. Mol. Biol. Cell 26, 4033-4045.

Sahl, S.J., Hell, S.W., and Jakobs, S. (2017). Fluorescence nanoscopy in cell biology. Nat. Rev. Mol. Cell Biol. 18, 685-701.

Schindelin, J., Arganda-Carreras, I., Frise, E., Kaynig, V., Longair, M., Pietzsch, T., Preibisch, S., Rueden, C., Saalfeld, S., Schmid, B., et al. (2012). Fiji: an open-source platform for biological-image analysis. Nat. Methods 9, 676-682.

Schneider, F., Waithe, D., Clausen, M.P., Galiani, S., Koller, T., Ozhan, G., Eggeling, C., and Sezgin, E. (2017). Diffusion of lipids and GPI-anchored proteins in actin-free plasma membrane vesicles measured by STED-FCS. Mol. Biol. Cell 28, 1507-1518.

Schneider, F., Waithe, D., Lagerholm, B.C., Shrestha, D., Sezgin, E., Eggeling, C., and Fritzsche, M. (2018). Statistical Analysis of Scanning Fluorescence Correlation Spectroscopy Data Differentiates Free from Hindered Diffusion. ACS Nano 12, 8540-8546.

Schneider, F., Hernandez-Varas, P., Christoffer Lagerholm, B., Shrestha, D., Sezgin, E., Julia Roberti, M., Ossato, G., Hecht, F., Eggeling, C., and Urbančič, I. (2020). High photon count rates improve the quality of super-resolution fluorescence fluctuation spectroscopy. J. Phys. D. Appl. Phys. 53, 164003.

Schwille, P., Meyer-Almes, F.J., and Rigler, R. (1997). Dual-color fluorescence cross-correlation spectroscopy for multicomponent diffusional analysis in solution. Biophys. J. 72, 1878-1886.

Sograte-Idrissi, S., Oleksiievets, N., Isbaner, S., Eggert-Martinez, M., Enderlein, J., Tsukanov, R., and Opazo, F. (2019). Nanobody Detection of Standard Fluorescent Proteins Enables MultiTarget DNA-PAINT with High Resolution and Minimal Displacement Errors. Cells 8, 48.

Sograte-Idrissi, S., Schlichthaerle, T., Duque-Afonso, C.J., Alevra, M., Strauss, S., Moser, T., Jungmann, R., Rizzoli, S.O., and Opazo, F. (2020). Circumvention of common labelling artefacts using secondary nanobodies. Nanoscale 818351.

Stanly, T.A., Fritzsche, M., Banerji, S., García, E., Bernardino de la Serna, J., Jackson, D.G., and Eggeling, C. (2016). Critical importance of appropriate fixation conditions for faithful imaging 
679 of receptor microclusters. Biol. Open 5, 1343-1350.

680 Sun, Y., Day, R.N., and Periasamy, A. (2011). Investigating protein-protein interactions in living 681 cells using fluorescence lifetime imaging microscopy. Nat. Protoc. 6, 1324-1340.

682 Suzuki, K.G.N., Kasai, R.S., Hirosawa, K.M., Nemoto, Y.L., Ishibashi, M., Miwa, Y., Fujiwara, 683 T.K., and Kusumi, A. (2012). Transient GPI-anchored protein homodimers are units for raft 684 organization and function. Nat. Chem. Biol. 8, 774-783.

685 Traub, L.M. (2019). A nanobody-based molecular toolkit provides new mechanistic insight into 686 clathrin-coat initiation. Elife 8, 1-42.

687 Tsien, R.Y. (1998). THE GREEN FLUORESCENT PROTEIN. Annu. Rev. Biochem. 67, 509688544.

689 Veerapathiran, S., and Wohland, T. (2017). The imaging FCS diffusion law in the presence of 690 multiple diffusive modes. Methods 140-141, 140-150.

691 Waithe, D., Clausen, M.P., Sezgin, E., and Eggeling, C. (2016). FoCuS-point: software for 692 STED fluorescence correlation and time-gated single photon counting. Bioinformatics 32, 958693960.

694 Waithe, D., Schneider, F., Chojnacki, J., Clausen, M.P., Shrestha, D., de la Serna, J.B., and 695 Eggeling, C. (2018). Optimized processing and analysis of conventional confocal microscopy 696 generated scanning FCS data. Methods 140-141, 62-73.

697 Weiß, K., Neef, A., Van, Q., Kramer, S., Gregor, I., and Enderlein, J. (2013). Quantifying the 698 diffusion of membrane proteins and peptides in black lipid membranes with 2-focus fluorescence 699 correlation spectroscopy. Biophys. J. 105, 455-462.

700 Widengren, J., Mets, U., and Rigler, R. (1995). Fluorescence correlation spectroscopy of triplet 701 states in solution: a theoretical and experimental study. J. Phys. Chem. 99, 13368-13379. 